\title{
Development of a novel nanoflow liquid chromatography- parallel reaction monitoring mass spectrometry-based method for quantification of angiotensin peptides in HUVEC cultures
}

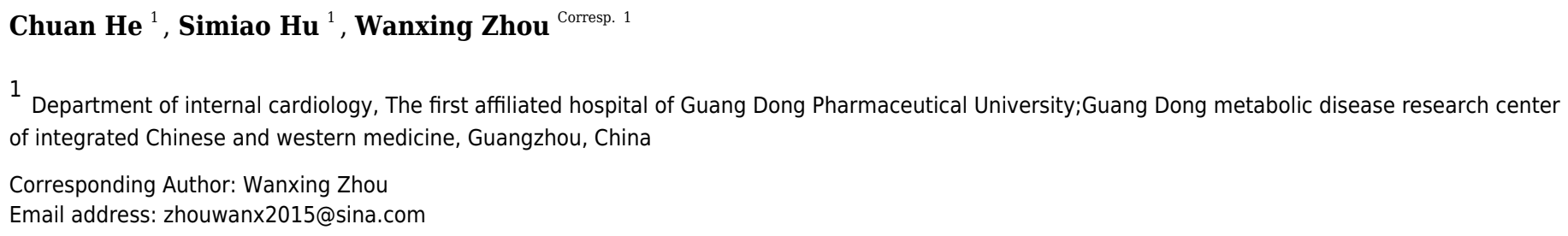

Background: This study aimed to develop an analytical method using liquid chromatography tandem mass spectrometry (LC-MS/MS) for the determination of angiotensin (Ang) I, Ang (1-9), Ang II, Ang (1-7), Ang (1-5), Ang III, Ang IV in human umbilical vein endothelial cell (HUVEC) culture supernatant. Methods: HUVEC culture supernatant was added with gradient concentrations $(0.05-1000 \mathrm{ng} / \mathrm{ml})$ of standard solutions of the Ang peptides. These samples underwent $\mathrm{C} 18$ solid-phase extraction and separation using a preconcentration nano-liquid chromatography mass spectrometry system. The target peptides were detected by a Q Exactive quadrupole orbitrap highresolution mass spectrometer in the parallel reaction monitoring mode. Ang converting enzyme (ACE) in HUVECs was silenced to examine Ang I metabolism. Results: The limit of detection was $0.1 \mathrm{pg}$ for Ang II and Ang III, and $0.5 \mathrm{pg}$ for Ang (1-9), Ang (1-7), and Ang (1-5). The linear detection range was 0.1-2000 pg (0.05-1000 ng/ml) for Ang II and Ang III, and $0.5-2000 \mathrm{pg}(0.25-1000 \mathrm{ng} / \mathrm{ml})$ for Ang (1-9) and Ang (1-5). Intra-day and inter-day precisions (relative standard deviation) were $<10 \%$. Ang II, Ang III, Ang IV, and Ang (1-5) were positively correlated with ACE expression by HUVECs, while Ang I, Ang (1-7), and Ang (1-9) were negatively correlated. Conclusion: The nanoflow liquid chromatographyparallel reaction monitoring mass spectrometry-based methodology established in this study can evaluate the Ang peptides simultaneously in HUVEC culture supernatant. 
1 Development of a novel nanoflow liquid chromatography-parallel reaction monitoring

2 mass spectrometry-based method for quantification of angiotensin peptides in HUVEC

3 cultures

4

5 Running title: Angiotensin quantification by nano-LC/PRM-MS in HUVECs

7 Chuan He, Simiao Hu, Wanxing Zhou*

8 Department of internal cardiology, The first affiliated hospital of Guang Dong Pharmaceutical

9 University; Guang Dong metabolic disease research center of integrated Chinese and western medicine; Guangzhou, China

\section{*Corresponding author}

13 Wanxing Zhou

Department of internal cardiology, The first affiliated hospital of Guang Dong Pharmaceutical

University; Guang Dong metabolic disease research center of integrated Chinese and western medicine; Guangzhou, China

Tel: $+86-13502404757$

Email: zhouwanx2015@sina.com

Fax: $+86-61327317$ 


\section{Abstract}

23 Background: This study aimed to develop an analytical method using liquid chromatography tandem mass spectrometry (LC-MS/MS) for the determination of angiotensin (Ang) I, Ang (1-9), Ang II, Ang (1-7), Ang (1-5), Ang III, Ang IV in human umbilical vein endothelial cell (HUVEC) culture supernatant.

Methods: HUVEC culture supernatant was added with gradient concentrations $(0.05-1000 \mathrm{ng} / \mathrm{ml})$ of standard solutions of the Ang peptides. These samples underwent C18 solid-phase extraction and separation using a preconcentration nano-liquid chromatography mass spectrometry system. The target peptides were detected by a Q Exactive quadrupole orbitrap high-resolution mass spectrometer in the parallel reaction monitoring mode. Ang converting enzyme (ACE) in HUVECs was silenced to examine Ang I metabolism.

Results: The limit of detection was $0.1 \mathrm{pg}$ for Ang II and Ang III, and $0.5 \mathrm{pg}$ for Ang (1-9), Ang (1-7), and Ang (1-5). The linear detection range was 0.1-2000 pg (0.05-1000 ng/ml) for Ang II and Ang III, and 0.5-2000 pg (0.25-1000 ng/ml) for Ang (1-9) and Ang (1-5). Intra-day and inter-day precisions (relative standard deviation) were $<10 \%$. Ang II, Ang III, Ang IV, and Ang (1-5) were positively correlated with ACE expression by HUVECs, while Ang I, Ang (1-7), and Ang (1-9) were negatively correlated.

Conclusion: The nanoflow liquid chromatography-parallel reaction monitoring mass spectrometry-based methodology established in this study can evaluate the Ang peptides simultaneously in HUVEC culture supernatant. 
43 Keywords: Angiotensin; high-resolution mass spectrometry; human umbilical vein endothelial 44 cells; parallel reaction monitoring; renin-angiotensin system. 
Introduction

48 The angiotensin (Ang) family is part of the renin-angiotensin system (RAS) and is involved in cardiovascular homeostasis and health by regulating the blood pressure (Basso and Terragno 2001). The family includes Ang I and its metabolites: Ang II, Ang III, Ang IV, Ang (1-9), Ang

51 (1-7), and Ang (1-5) (Supplementary Figure S1). Ang I is hydrolyzed by the Ang converting enzyme (ACE) to form Ang II (Culver et al. 2017). Ang II can induce intense arterial vasoconstriction and elevate blood pressure by activating the Ang type 1 receptor (AT1), induce endothelial dysfunction, and the pathological remodeling of cardiovascular tissue and aggravate water and sodium retention and cardiovascular remodeling by up-regulating aldosterone (Touyz 2003). Ang I can also be hydrolyzed by the Ang converting enzyme 2 (ACE2) to form Ang (1-9), which is further hydrolyzed by ACE to form Ang (1-7) and Ang (1-5). Ang (1-9) can bind to the Ang type 2 receptor (AT2) and block Ang II-induced cardiac hypertrophy. Meanwhile, it can serve as a substrate for ACE, reducing Ang II production through competition with Ang I (Buczko et al. 2006). Ang (1-7) modulates the nitric oxide release from endothelial cells through the Mas receptors (Santos and Ferreira 2007) and inhibits sodium reabsorption in the kidney

(Castelo-Branco et al. 2013). In addition, Ang II can be hydrolyzed into Ang III, which has effects similar to Ang II (Kemp et al. 2012). Ang III can be further hydrolyzed to generate Ang IV, which can activate the nuclear factor (NF)- $\mathrm{kB}$ to up-regulate the expression of interleukin (IL)6 and tumor necrosis factor (TNF)- $\alpha$, which promote the proliferation and migration of vascular endothelial cells and smooth muscle cells, leading to cardiovascular remodeling (Fyhrquist and 
67 Saijonmaa 2008). Hence, Ang metabolism is complex, and each member has specific biological

68

69

functions, and they can interact with each other. Due to the large span and low content of Ang peptides in different body substrates (blood, urine, pleural effusion, and various tissues), great differences in concentrations of each Ang are observed (Lortie et al. 2009). Therefore, a method with high throughput, wide linear dynamic range, high sensitivity, and high reproducibility is needed. The traditional methods for detecting Ang peptides are immunological methods (Chappell et al. 2012; Chappell 2016). In addition to the need for developing the antibodies, these methods have lower detection throughput, narrow linear dynamic range, and immune cross-reactivity among Ang metabolites because they share the same amino acid sequences (Jankowski et al. 2011).

Compared with immunological methods, liquid chromatography-mass spectrometry (LC-MS) directly analyzes the molecular structures, avoiding the need for antibody preparation. It has extremely high specificity and retains linearity over a wide concentration range (Olkowicz et al. 2017; Tamvakopoulos 2007). It can simultaneously detect multiple components in one sample. Liquid chromatography tandem mass spectrometry (LC-MS/MS) can be applied for the quantitative analysis of polypeptides. The new high-resolution mass spectrometer Q Exactive used in the present study can reach a resolution of 140,000 (Eliuk and Makarov 2015). Parallel reaction monitoring (PRM) was developed on the basis of select reaction monitoring (SRM). PRM is based on a quadrupole high-resolution mass spectrometer platform that included the QOrbitrap device. Unlike the SRM that only select one specific fragment ion for detection and analysis in the secondary mass spectrometry, the PRM collects all the fragment ions after the 
fragmentation of the precursor ion, resulting in better reliability and specificity (Malchow et al. 2017; Ronsein et al. 2015; Bourmaud et al. 2016). The first step of PRM is to use the quadrupole (Q1) to select the parent ion. The second step is to break the parent ion in the collision pool (Q2) to form the daughter ions. Finally, the Orbitrap is used to replace Q3, and all the sub-ions are scanned in the high-resolution/high-quality precision (HR/AM) mode to complete PRM data acquisition (Gallien et al. 2013). Compared with the previous SRM/multiple reaction monitoring (MRM) used in quantitative Ang analysis, the PRM selected in this study has some advantages: 1) high-resolution sub-ion monitoring, quality accuracy up to the part-per-million level, and eliminating background interference in cell culture matrix to the greatest extent without loss of sensitivity; 2) two-stage mass spectrometry within a single full scan, which requires no prior determination of ion pairs and optimization of collision energy; only one or several sub-ions with the highest response can be selected during data processing to extract the chromatographic peaks of the parent and child ion pairs for quantitative analysis, with a linear range of 5-6 orders of magnitude; and 3) both qualitative and quantitative analyses are performed simultaneously. The second-level full scan spectrum is used for qualitative analysis, and the optimal sub-ion extraction ion pair can be used for quantitative analysis. High-throughput analysis of multiple Ang peptide data can save a lot of time in matching ion pairs and parameter optimization.

Human umbilical vein endothelial cells (HUVECs) have become an important model for studying vascular physiological functions and pathological remodeling (Nachman and Jaffe 2004). Although previous studies reported the use of the SRM LC-MS/MS technique to detect the Ang peptides in blood, urine, and tissues (Olkowicz et al. 2017; Olkowicz et al. 2015; Ali et 
109

110

111

112

113

114

115

116

117

118

119

120

121

122

123

124

125

126

127

128

129

8

al. 2014), the methods for detecting the peptides in cell culture supernatants were rarely reported(Finoulst et al. 2011a; Finoulst et al. 2011b; Kay et al. 2018). Culture media often contain substances that are not found in body fluids and tissues, and lowly-expressed peptides may be masked by more abundant ones, resulting in potential interference. In addition, there is a lack of studies for the mass spectrometry detection of Ang peptides using the PRM model.

We hypothesized that the PRM LC-MS/MS technique could be used to detect Ang peptides from HUVEC culture supernatants. Therefore, the present study aimed to establish a set of methods for the quantitative analysis of Ang peptides in HUVEC culture supernatants, more specifically Ang I, Ang (1-9), Ang II, Ang (1-7), Ang (1-5), Ang III, and Ang IV.

\section{Materials and methods}

\section{Experimental design}

This study was conducted in two phases. In the first phase, the LC-MS/MS protocol was optimized using culture supernatant. In the second phase, in order to examine whether the detection method was sensitive to changes in Ang metabolism, ACE was silenced in cells, and the Ang profile was measured.

\section{Reagents}

The reagents are presented in the Supplementary Data.

\section{Cell lines}

The human umbilical vein endothelial cells (HUVEC) were bought from ScienCell Research Laboratories (USA, Catalog Number: 8000, Lot Number: 14260). They were primary cells at 
130

131

132

133

134

135

136

137

138

139

140

141

142

143

144

145

146

147

148

149

150

passage 0 .

\section{Optimization of the LC-MS/MS conditions and establishment of the PRM methodology}

The pieces of equipment are presented in the Supplementary Data.

Ang I, Ang (1-7), Ang II, Ang (1-9), Ang (1-5), Ang III, and Ang IV standard solutions and internal standard solutions were prepared in $0.1 \%$ formic acid water solution at $1 \mu \mathrm{g} / \mathrm{ml}$ of the Ang peptide. The solutions $(2 \mu \mathrm{l})$ were loaded at $5 \mu \mathrm{l} / \mathrm{min}$. Mobile phase A was $0.1 \%$ formic acid water solution. Mobile phase B was $0.1 \%$ formic acid acetonitrile solution. The elution gradient was $2 \% \mathrm{~B}$ at $0 \mathrm{~min}, 4 \% \mathrm{~B}$ at $2 \mathrm{~min}, 22 \% \mathrm{~B}$ at $47 \mathrm{~min}, 35 \% \mathrm{~B}$ at $57 \mathrm{~min}, 90 \% \mathrm{~B}$ at $62 \mathrm{~min}$, and $92 \% \mathrm{~B}$ at $70 \mathrm{~min}$. The flow rate was $250 \mathrm{nl} / \mathrm{min}$.

The nano-ESI was combined with a positive ion mode. Spray voltage was $2.25 \mathrm{kV}$. The capillary temperature was $320^{\circ} \mathrm{C}$. The S-lens was $50 \%$. The full scan $+\mathrm{ddMS}^{2}$ mode was used.

Resolution setting were full scan 70,000 and ddMS 2 17,500. The precursor ion scan range was $\mathrm{m} / \mathrm{z}$ 250-1800. The collision energy was $27 \%$ HCD (high energy collision dissociation). The acceptable automatic gain control (AGC) target was full scan, and $3 \mathrm{e} 6$ and the ddMS ${ }^{2}$ was 5 e4.

The maximum injection time (maximum IT) was full scan, $60 \mathrm{~ms}$, and the ddMS ${ }^{2}$ was $80 \mathrm{~ms}$. The Skyline Ang spectra library was constructed based on the amino acid sequence of the peptides. The detected mass spectral data were searched through a database, and the best precursor ion of the target peptide was selected for establishing the method.

The precursor ion mass-to-charge ratio $(\mathrm{m} / \mathrm{z})$ of the target peptide was added to the inclusion list to establish a mass spectrometry acquisition method. PRM data of the sample was acquired using the PRM method and to determine the final PRM method for sample data collection. The 
151

152

153

154

155

156

157

158

159

160

161

162

163

164

165

166

167

168

169

170

171

resolution was MS: 70,000. The MS/MS ratio was 35,000. The precursor ion scan range was $\mathrm{m} / \mathrm{z}$ 300-1500. The AGC target was 3e6 for MS and 2e5 for MS/MS. The maximum IT was $60 \mathrm{~ms}$ for MS and $100 \mathrm{~ms}$ for MS/MS. The fixed first mass was $100 \mathrm{~m} / \mathrm{z}$. The isolated window was 1.6 $\mathrm{m} / \mathrm{z}$. The MS information about the Ang family is presented in Supplementary Table S4.

\section{HUVEC culture and preparation of cell supernatant matrix solution}

HUVECs were inoculated in culture flasks and grown to $70 \%$ confluence. The original culture medium was discarded. The cells were washed three times with phosphate buffer saline (PBS).

Phenol red-free endothelial cell basal medium (ECM-prf) $(5 \mathrm{ml})$ and 1\% endothelial cell growth supplements (ECGS) were added, and the cells were cultured at $37^{\circ} \mathrm{C}$ with $5 \% \mathrm{CO}_{2}$ for $24 \mathrm{~h}$. Culture supernatants were harvested and centrifuged at $2000 \mathrm{~g}, 4^{\circ} \mathrm{C}$, for $15 \mathrm{~min}$. A mass spectrometry-specific protease inhibitor mixture (Beyotime Biotechnology, China) and $50 \mathrm{mM}$ ethylene diamine tetraacetic acid (EDTA) solution were added at 1:50, and $576 \mathrm{mM} 2$ mercaptoethanol (Sigma, St Louis, MO, USA) was added at 1:40. The final cell supernatant solution was stored at $-80^{\circ} \mathrm{C}$.

\section{Development and validation of the methodology}

\section{Evaluation of linear dynamic range and sensitivity}

Trifluoroacetic acid (TFA) $(2.5 \%)$ was added to the HUVEC culture supernatant solution to achieve a final TFA concentration of $0.2 \%$. After centrifugation at $12,000 \mathrm{~g}, 4^{\circ} \mathrm{C}$, for $30 \mathrm{~min}$, the supernatant was mixed with the standard peptide solution to prepare a gradient concentration of 10 samples: $1000,500,100,20,10,5,1,0.5,0.25$, and $0.05 \mathrm{ng} / \mathrm{ml}$. The internal standard $\mathrm{Val}_{5^{-}}$ Ang II was $100 \mathrm{ng} / \mathrm{ml}$. 
TFA. After dilution, the sample was loaded on the equilibrated SPE cartridge at $3 \mathrm{ml} / \mathrm{min}$. The sample passed through the bed of the cartridge that was collected and loaded twice, and then washed three times with $5 \%$ methanol, $1 \mathrm{ml} /$ time. The cartridge underwent continuous vacuum drying for 5 min to allow the bed to be completely dry. Then, $80 \%$ acetonitrile $/ 0.1 \%$ TFA was used for three elutions, with $500 \mathrm{ml}$ each. The eluent was collected in centrifuge tubes. The

'Concentrator plus' vacuum centrifugation concentrator (Eppendorf, Hamburg, Germany) was used for vacuum centrifugation and swab-off of the samples.

The $0.1 \%$ formic acid $/ 2 \%$ acetonitrile solution $(100 \mu \mathrm{l})$ was used for re-dissolution, and $2 \mu 1$ of the sample was loaded. The ratio and the theoretical content were used to calculate the correlation coefficient, slope, and intercept of the linear regression equation, and the standard curve was plotted. The lower limit of quantitation (LLOQ) was a signal-to-noise ratio $(\mathrm{S} / \mathrm{N}) \geq 10$.

Evaluation of precision

The mixed Ang peptide solution at a concentration of $10 \mathrm{ng} / \mathrm{ml}$ was selected for quality control (QC). For intra-day precision evaluation, QC samples were repeatedly tested five times on the same day. For inter-day precision evaluation, QC samples were pre-processed in the same way and repeatedly tested for 3 days. The detection precision was assessed by the intra-day and inter-day relative standard deviation (RSD). $\mathrm{RSD}=$ (standard deviation of Ang peptide 
193

194

195

196

197

198

199

200

201

202

203

204

205

206

207

208

209

210

211

212

213

area) $* 100 \%$.

\section{Sample stability}

The minimum operating temperature of the liquid sample injector was $7^{\circ} \mathrm{C}$. All samples were stored at $-80^{\circ} \mathrm{C}$ and prepared in batches of groups of three samples before the test. The test time of each sample was 80 minutes, and the total operation time for one group was $4 \mathrm{~h}$. QC samples were tested on the machine after pretreatment and repeated six times. Percentage of sample stability $=$ (peak area of quantitative fragment ions for Ang in loading sample/peak area of quantitative fragment ions for Ang in the first loading sample) $* 100 \%$. A percentage of $85 \%$ $115 \%$ was considered stable.

\section{Effect of pretreatment on sample detection}

\section{Effect of solid-phase extraction column loading frequency on angiotensin detection}

The number of repeated loading on the SPE cartridge was evaluated using two, three, or four times. The QC sample solution $(100 \mu \mathrm{l})$ was diluted with $400 \mu \mathrm{l}$ of $0.1 \%$ TFA. The column solution was collected after the sample was passed through the SPE cartridge. The samples were detected to compare the differences in the detection value of Ang peptides after different numbers of SPE treatments.

\section{Effect of ultrafiltration on angiotensin peptide analysis}

In the SPE group, $100 \mu \mathrm{l}$ of the QC sample solution was diluted with $400 \mu \mathrm{l}$ of $0.1 \% \mathrm{TFA}$. The sample was loaded on the bed of the cartridge three times, according to the above method. For the ultrafiltration centrifugation + SPE group, ultrafiltration centrifugation was first performed to remove the proteins; $100 \mu \mathrm{l}$ of the QC sample solution was added to the inner cannula of an 
214 ultrafiltration tube (MWCO $10 \mathrm{kDa}$, PES, Pall Life Sciences, Ann Arbor, MI, USA) and 215 centrifuged at $4^{\circ} \mathrm{C}, 12,000 \mathrm{rpm}$ for $10 \mathrm{~min}$. Then, $200 \mu \mathrm{l}$ of the $0.1 \%$ TFA solution was added to 216 the inner cannula for rinsing. It was centrifuged and then rinsed and centrifuged. The liquid in 217 the outer cannula was collected. Then, the above SPE operation was performed again.

218 The samples in the two groups were tested after SPE to compare the difference in the detection 219 value of Ang peptides in the two groups and evaluate the effect of ultrafiltration on the Ang peptide analysis in samples.

\section{Effect of different ACE-expressing HUVECs on Ang I metabolism}

The RNA interference technique was used to construct model cell lines with lentivirus-based

shRNA interference sequences to stably silence ACE expression in HUVECs. The construction

method and results are shown in the Supplementary Material. The HUVEC model group with a

negative sequence introduction and normal ACE expression was the PLKO.1 group. HUVECs

with the ACE-shRNA interference sequence introduction and stable silencing of ACE gene

expression were the interference group. It was divided into the ACE-shRNA-1 and ACE-

shRNA-2 group according to $99 \%$ and $73 \%$ downregulation of ACE mRNA levels. See the

Supplementary Data for the methods regarding the detection of Ang I and its metabolites.

\section{Data analysis}

Skyline was used to import the mass spectrometric data files. The three strongest precursor ion

$232(\mathrm{M}, \mathrm{M}+\mathrm{H}$, and $\mathrm{M}+2 \mathrm{H})$ peaks for each peptide were extracted. The ion match tolerability was

$0.01 \mathrm{~m} / \mathrm{z}$, satisfying idopt $\geq 0.9$. The eight strongest $\mathrm{y}, \mathrm{b}$ fragment ion peaks for each peptide were extracted. One of the quantitative fragment ion, complex ion, precursor ion match 
235 information, and retention time with high specificity and intensity was selected for qualitative

236 analysis of Ang metabonomics. The relative quantitative analysis was performed using the ratio

237 of Ang peptide fragment ion area to the internal standard fragment ion area. The standard curve

238 method was used for absolute quantitative analysis of Ang. The t-test was used to analyze the

239 differences between the two groups. Differences among the three groups were compared using one-way ANOVA. $P<0.05$ was considered statistically significant. The CORREL function in MS

Excel (Microsoft, Redmond, WA, USA) was used to analyze the correlations between the two sets of data and plot the correlation matrix.

\section{Results}

The LC-MS/MS parameters were optimized, and the PRM detection model was established.

The seven target peptides and the internal standard peptide could complete the column elution with acetonitrile concentrations of $10 \%-16 \%$. In order to separate the elution peaks and not affect the peak shape, the separation stages were set using $4 \% \mathrm{~B}$ at $2 \min$ to $22 \% \mathrm{~B}$ at $47 \mathrm{~min}$. The peaks of the target peptide and internal standard peptide were smooth and separated (Figure 1). The optimal sample dilution and loading volume were determined according to the different peak concentrations of the sample as $100 \mu \mathrm{l}$ for re-dissolution and loading volume of $2 \mu \mathrm{l}$.

Mass spectrometry parameters were optimized by tuning the capillary voltage, collision energy, and ion dwell time. At a capillary voltage of $2.25 \mathrm{kV}$, the $(\mathrm{M}+3 \mathrm{H})$ precursor ions of Ang I and Ang (1-9) were mainly the charged precursor ions, and the remaining of the target peptides 
256 established based on these results, and the ion dwell time was adjusted according to the number

257 of precursor ions. Using the peptide map library pre-built using Skyline, the fragment ions in the

258 PRM scan data were extracted (Figure 2). The fracture law and abundance of fragment ions were

259 matched with the spectrum library. The mass spectral response was up to $10^{6}$ orders of

260 magnitude. The signal-to-noise ratio was high, and the mass spectral peak shape was smooth.

261 The standard curves were built, and the detection limits were determined

262 The linear correlation coefficients of the standard curves were analyzed using least-squares

263 regressions and were all $\geq 0.99$ (Supplementary Figure S2), suggesting high detection accuracy.

264 The results of the slope, intercept, linear range, and the LLOQ of the LC-MS/MS linear detection

265 (Supplementary Table S5) showed that the linear dynamic ranges of most Ang peptides reached

$2662-4$ orders of magnitude, and the LLOQ reached among $0.05-0.25 \mathrm{ng} / \mathrm{ml}$. This method had a wide

267 linear dynamic range and high sensitivity and could satisfy the detection of multiple Ang

268 peptides with large differences in concentration.

269 The precision was determined

270 The intra-day precision of the seven Ang peptides in the QC sample was RSD $<10 \%$, and the

inter-day precision was also RSD $<10 \%$ (Supplementary Table S6). All errors were within the

allowable range, indicating that this method had high repeatability and optimal stability in

detecting the Ang metabolites in the cell culture supernatant.

The sample stability was determined

275 The Ang peptides were stable at $7^{\circ} \mathrm{C}$ in the liquid sample injector for $6 \mathrm{~h}$, and the 
277

278

279

280

281

282

283

284

285

286

287

288

289

290

291

292

293

294

295

296

297

\section{The loading times did not affect sample recovery}

We used three loadings on the cartridge as control and evaluated the other numbers of loading times on the recovery rate of Ang by comparing the peak area ratio of the same target peptide quantitative fragment ion. There were no significant differences in the recovery rate among different loading times (all $\mathrm{CV}<11.9 \%$, Figure 4), suggesting that the recovery rate was stable when the sample was loaded on the cartridge for two, three, or four times.

\section{Ultrafiltration reduced sample recovery}

The peak area of the Ang fragment ion in the ultrafiltration and SPE group was used as a normalization factor. The recovery rate of Ang I in the SPE group was significantly higher (3.91 times) than in the ultrafiltration and SPE group. The recovery ratios of Ang (1-9), Ang II, and Ang III in the SPE group were higher than in the ultrafiltration and SPE group $(1.20,1.42$, and 1.35 times high, respectively). There were no significant differences in the recovery rates of other target peptides between the two groups $(\mathrm{CV}<10 \%$, Figure 5). This indicated that ultrafiltration would significantly reduce the recovery rate of Ang I, as well as Ang II, Ang III, and Ang (1-9).

\section{ACE protein expression was successfully silenced in HUVECs}

The mRNA results show that the two interfering sequences caused the ACE mRNA to be down-regulated by 73\% and 99\% (Supplementary Table S3, Supplementary Figure S3). Western blotting showed that the expression of the ACE protein in the ACE-shRNA-1 and ACE-shRNA2 groups was significantly reduced (95.6\% and 78.2\%, respectively) compared with the PLKO.1 group (Figure 6). 
298

299

300

301

302

303

304

305

306

307

308

309

310

311

312

313

314

315

316

317

318

ACE silencing induces differences in the Ang metabolic profile that can be detected by LCMS/MS

To verify the practicability of the Ang assay established in this study, we added Ang I at a final concentration of $1 \mu \mathrm{M}$ to three groups of HUVECs with different ACE expression levels. After incubation for $1 \mathrm{~h}$, Ang I and its major metabolites (Ang II, Ang (1-7) and Ang (1-5), Ang (1-9), Ang III, and Ang IV) were detected by the LC-MS/MS method established in this study. The Ang II, Ang III, Ang IV, and Ang (1-5) levels in the ACE-shRNA-1 and ACE-shRNA-2 groups were significantly reduced compared with that in the PLKO.1 group. The decrease in the ACE-shRNA-1 group was more obvious than in the ACE-shRNA-2 group. Ang (1-9) and Ang (1-7) levels were significantly elevated, and the increase of Ang (1-9) in the ACE-shRNA-2 group was more obvious than in the ACE-shRNA-1 group. Ang (1-7) had an increasing trend. The Ang I concentrations in the ACE-shRNA-1 and ACE-shRNA-2 groups were significantly higher than in the PLKO.1 group (Figure 7). Ang I, Ang (1-9), and Ang (1-7) concentrations in the culture supernatant of HUVECs were significantly and negatively correlated with the expression of ACE. The Ang II, Ang III, Ang IV, and Ang (1-5) concentrations in the culture supernatants showed significant positive correlations with ACE protein expression (Supplementary Figure S4).

\section{Discussion}

In the present study, the slope, intercept, linear range, and the LLOQ of the LC-MS/MS linear detection showed that the linear dynamic ranges of most Ang peptides reached 2-4 orders of 
319 magnitude, and the LLOQ reached the level of the femtogram. Previous studies using LC-

$320 \mathrm{MS} / \mathrm{MS}$ to detect Ang peptides in biological samples also examined the linear dynamic range and

321 quantitation limits of the method. Cui et al. (Cui et al. 2007) detected Ang II, Ang III, Ang IV,

322 and Ang (1-7) in a bovine endothelial cell culture system; the linear dynamic range was 50-1250

323 pg on the column, concentration, and injection volume unknown, one order of magnitude, and

324 the limit of detection was $25 \mathrm{pg}$ on the column. Suski et al. (Suski et al. 2014) established a

method for the detection of Ang II and Ang (1-7) in a rat smooth muscle cell culture system; the

linear dynamic range was $2.5-250 \mathrm{ng} / \mathrm{ml}$ (about $5-500 \mathrm{pg}$ on the column, linear coefficient

unknown), with two orders of magnitude. Bujak-Gizycka et al. (Bujak-Gizycka et al. 2007)

detected the metabolites of Ang I in an EA.hy926 cell culture system; the dynamic linear range

was $20 \mathrm{pM}-100 \mathrm{nM}$ (about $0.02 \mathrm{ng} / \mathrm{ml}-100 \mathrm{ng} / \mathrm{ml}, 0.1-500 \mathrm{pg}$ on the column, MW was set in 1

$\mathrm{kD}$, linear coefficient unknown), with three orders of magnitude.

In the present study, the Ang peptides were stable at $7^{\circ} \mathrm{C}$ in the liquid sample injector for $6 \mathrm{~h}$,

and the concentration change was $<15 \%$. Previous studies showed that the optimal Ang storage

conditions in serum samples were $-80^{\circ} \mathrm{C}$ for 1 month, repeated freeze-thaw for three times, and

room temperature for $3 \mathrm{~h}$ (Olkowicz et al. 2017; Olkowicz et al. 2015). Ang I and metabolites

can remain stable for $12 \mathrm{~h}$ at $4{ }^{\circ} \mathrm{C}$ in an autosampler with a loss of $<15 \%$. To increase the stability

of the sample and reduce the loss of Ang peptides, the following points may be noted. First, a

low-temperature operation is required during collection, and various protease inhibitors should

be added in time to fully inhibit the proteases involved in Ang metabolism (Olkowicz et al. 2017;

Olkowicz et al. 2015). In addition, sample pretreatment should be completed as fast as possible. 
340 The samples that cannot be promptly pre-treated should be quick-frozen in liquid nitrogen and

341 stored at $-80^{\circ} \mathrm{C}$. It should be rapidly thawed at room temperature before use to avoid the recovery

342 of protease activity caused by slow warming. Second, the sample should be analyzed as soon as

343 possible after the completion of the pretreatment process. It has been reported that Ang peptides

344 are lost during long-term storage regardless of the conditions (Lortie et al. 2009; Olkowicz et al.

345 2015; Ali et al. 2014). It is related to the metabolic activities of enzymes in biological matrixes

346 and material adsorption in storage containers. For some samples that cannot be immediately

347 tested, they can be vacuum-dried and stored at $-80^{\circ} \mathrm{C}$. Third, the sample bottles used for analysis

348 were surface-silicified or polypropylene micro-injection bottles pre-coated with a saturated

349 peptide mixture were used, avoiding the use of conventional glass insert-pipes. Small volumes

350 dissolution was performed before loading on the machine to reduce the absorption of Ang

351 peptides by the sample containers (Lortie et al. 2009; Stejskal et al. 2013).

352 Ultrafiltration is a commonly used method for sample pretreatment before LC-MS to separate

proteins and small molecules. It is expected to increase the purity of the sample. The exfoliated

cells, cell debris, other particulate-like substances, and some large proteins in the cell supernatant

can be removed during ultrafiltration, leaving small peptides. In addition, proteases can be

removed, increasing sample stability. On the other hand, ultrafiltration may also cause an

unexpected loss of Ang peptides. Some authors have used ultrafiltration to purify serum and used

LC-MS to detect Ang I and Ang II (Lortie et al. 2009; Chappell et al. 2012), but they did not

evaluate the impact of ultrafiltration on the recovery rate of Ang in cell culture supernatants. The 
361 the basic peptide and the negatively charged proteins and the hydrophobic interaction between

362 the hydrophobic peptides and the materials of the ultrafiltration tube and filter membrane. The

363 ultrafiltration tube lining used in this study was made of polypropylene, and the ultrafiltration

364 membrane was made of polyethersulfone. It has been reported that polyethersulfone can absorb a

365 large number of polypeptide molecules with nonpolar side chains. As a result, polypeptide

366 molecules were trapped, and the recovery rate was reduced (Hu and Kamberi 2009). Ang I, Ang

367 (1-9), Ang II, and Ang III have phenylalanine at their carboxyl terminus, with strong

368 hydrophobicity. Adding a certain proportion of surfactant or nonpolar solvent, or using

369 acetonitrile to reduce the polarity of the cell supernatant in the sample solution, would reduce the

370 adsorption of Ang peptide by the ultrafiltration materials (Hu and Kamberi 2009). Selecting an

371 ultrafiltration membrane and ultrafiltration tube with low adsorption materials (such as cellulose

372 acetate) may also improve its recovery rate. An acid or a base could be added to the collected

373 samples so that the proteins and polypeptides have the same charges in order to reduce

374 electrostatic adsorption (Che et al. 2010).

375 The eluted sample after SPE was finally dried by using a vacuum centrifugal concentrator to

remove the elution solvent. It reduced the contamination and loss among samples compared with

377 the nitrogen blowing method and was relatively simple and economical compared with the

freeze-drying method. In the view of the experiences by others and our study, the following

points may be the key to improve the pretreatment of the samples for the detection of Ang

peptides in cell supernatants using LC-MS. First, the cell culture medium was routinely added

with phenol red as an acid-base indicator. In our previous work, we found that phenol red was a 
382 high-retention material in the chromatographic system and would severely interfere with the 383 purification and separation of Ang. Therefore, a phenol red-free medium is suggested for the use 384 of LC-MS. Secondly, Ang peptides have biological activity and are sensitive to microorganisms. 385 Therefore, the collection and pretreatment of samples should be conducted with strictly aseptic conditions. Thirdly, the addition of TFA to the sample for acidification can reduce the adsorption between basic Ang peptides and negatively charged macromolecular proteins in neutral solutions, promoting protein denaturation and precipitation (Chertov et al. 2004). On the other hand, acting as a strong ion pair reagent, TFA can improve the retention of Ang peptides in the C18 chromatographic columns. Fourthly, there were a lot of exogenously added amino acids and peptides in the cell culture medium, which would compete with the Ang peptides for the adsorption of the C18 column. The overloading phenomenon occurred when we used the micro SPE tips (Ziptip, Millipore, USA) to process the 100- $\mu$ l sample in our previous study. Therefore, the particularities of cell culture supernatant should be fully considered, and a high-load SPE cartridge should be selected.

ACE is a key metabolic enzyme in the RAS and is involved in multiple metabolic pathways of Ang (Culver et al. 2017). Ang II is generated by ACE from Ang I, and Ang III and Ang IV are downstream metabolites of Ang II. Ang (1-5) is generated by ACE from Ang (1-7). Ang I, Ang (1- 9), and Ang (1-7) are regulated by both ACE and ACE2 (Supplementary Figure S1). Therefore, as supported by the known metabolism of Ang (Streatfeild-James et al. 1998; Morris et al. 2020; Yugandhar and Clark 2013; Chai et al. 2004; Jackman et al. 2002; Yu et al. 2016), 
403

404

405

406

407

408

409

410

411

412

413

414

415

416

417

418

419

420

421

422

423

ACE expression was inhibited. The down-regulation of ACE expression inhibited the Ang I-

ACE-Ang II pathway, and the Ang I concentration was higher than in the normal ACE expression group. In this study, there was a significant difference in ACE expression levels between the ACE-shRNA-1 group and the ACE-shRNA-2 group, but the Ang I levels were not significantly different between the two groups. This may be related to an increase in ACE2 expression after down-regulation of ACE (Zhong et al. 2010). In addition, the activity of the Ang I-NEP/MP/E-Ang (1-7) pathway could be potentiated. The upregulation of these two pathways can increase Ang I catabolism. Therefore, it can explain the similar Ang I levels in different groups with different degrees of ACE inhibition. Down-regulation of the Ang (1-7)-ACE-Ang (1-5) pathway, the weakening of Ang (1-7) catabolism also increased the Ang (1-7) levels and decreased the Ang (1-5) levels. It can be seen that the down-regulation of ACE not only interfered with one metabolic pathway but also affected the entire Ang metabolic network.

Due to the low-quality resolution, it is difficult for MRM to remove the interference of complex matrix background effectively, and MRM is prone to false positive (Sherman et al. 2009; Abbatiello et al. 2010). On the other hand, with the increasing requirement of analytical flux, multiple ion pairs may need to be monitored in one analysis, while the limitation of MRM speed and sensitivity makes the number of ion pairs that can be monitored at the same time limited (Kiyonami et al. 2011). In addition, the optimization of ion pair, collision energy, and other conditions is time-consuming and laborious, which is difficult to meet the need for multiple product monitoring for metabolic network analysis (Kiyonami et al. 2011; Cima et al. 2011). Unlike the MRM approach, the quantitative fragment ions were not screened before the detection 
424 in the present study. We only first performed qualitative analysis using all the fragment ions with

425 the help of the Skyline mass spectrometry analysis software in the later stage. During the

426 quantitative analysis, we selected a fragment ion with a high signal as the quantitative fragment

427 ion, which saved a great deal of method development time compared with the MRM method,

428 while the sensitivity reached $0.1-0.5 \mathrm{pg}$ on the column. It demonstrated the superior qualitative

429 and quantitative capabilities of preconcentration nano-liquid chromatography coupled with

430 quadrupole electrostatic field orbitrap mass spectrometry. Secondly, in this study, the biological

431 matrix was the supernatant from HUVECs cultured in the endothelial cell culture medium, which

432 could be used continuously for cell culture. Therefore, the method established has a more

433 extensive application value.

434 In this study, a homologous internal standard was selected, in which valine was used to

435 replace the $5^{\text {th }}$ isoleucine of Ang II, with the remaining amino acid sequence being unchanged.

436 The internal isotopic standard is the gold standard for absolute quantification using LC-MS, but

437 the isotopic internal standard was not used in this study based on two considerations. First, this

438 study does not rely on the concentration of internal isotopic standard for the absolute

439 quantification of samples, and the internal standard is only used as the homogenization standard

440 for pretreatment to correct the errors caused by pretreatment. Second, homologous internal

441 indices were also used in the literature (Olkowicz et al. 2017; Olkowicz et al. 2015;

442 Tyrankiewicz et al. 2018). Undoubtedly, a stable isotopic internal standard is the most accurate

443 choice, but its preparation cost is high, and access to isotopes (including stable isotopes) is

444 limited in China. Due to limited funding, the homologous internal standard was used. 


\section{Conclusions}

447 The nano-liquid chromatography mass spectrometry with parallel reaction monitoring 448 established in this study can simultaneously and accurately detect multiple Ang peptides in the 449 culture supernatant of HUVECs. This method has the advantages of high sensitivity, wide linear 450 dynamic range, large throughput, and high efficiency. It is an advanced and reliable method for 451 the study of cellular metabonomics of Ang. This method may be an innovative method for 452 assessing the effect of RNA interference or therapeutic effects of ACE inhibitors, providing new 453 ideas and targets for the role of RAS in the research and treatment of hypertension and 454 cardiovascular remodeling.

455 Acknowledgments

456 None.

457 Funding

458 This work was supported by the science and technology plan of Guangdong provincial science 459 and technology department (grant number 2017ZC0204).

460 Conflict of Interest

461 The authors declare that they have no conflict of interest.

\section{References}

Abbatiello SE, Mani DR, Keshishian H, Carr SA (2010) Automated detection of inaccurate and imprecise transitions in peptide quantification by multiple reaction monitoring mass 
466 spectrometry. Clin Chem 56 (2):291-305. doi:10.1373/clinchem.2009.138420

467 Ali Q, Wu Y, Nag S, Hussain T (2014) Estimation of angiotensin peptides in biological samples

468 by LC/MS method. Analytical methods : advancing methods and applications 6 (1):215-222.

469 doi:10.1039/C3AY41305E

470 Basso N, Terragno NA (2001) History about the discovery of the renin-angiotensin system.

471 Hypertension 38 (6):1246-1249

472 Bourmaud A, Gallien S, Domon B (2016) Parallel reaction monitoring using quadrupole-

473 Orbitrap mass spectrometer: Principle and applications. Proteomics 16 (15-16):2146-2159.

474 doi:10.1002/pmic.201500543

475 Buczko W, Kramkowski K, Mogielnicki A (2006) Are the endothelial mechanisms of ACE-Is

476 already established? Pharmacol Rep 58 Suppl:126-131

477 Bujak-Gizycka B, Madej J, Wolkow PP, Olszanecki R, Drabik L, Rutowski J, Korbut R (2007)

478 Measurement of angiotensin metabolites in organ bath and cell culture experiments by liquid 479 chromatography - electrospray ionization - mass spectrometry (LC-ESI-MS). Journal of 480 physiology and pharmacology : an official journal of the Polish Physiological Society 58

$481 \quad(3): 529-540$

482 Castelo-Branco RC, Leite-Delova DC, de Mello-Aires M (2013) Dose-dependent effects of angiotensin-(1-7) on the NHE3 exchanger and $[\mathrm{Ca}(2+)](\mathrm{i})$ in in vivo proximal tubules. Am $\mathrm{J}$ Physiol Renal Physiol 304 (10):F1258-1265. doi:10.1152/ajprenal.00401.2012

485 Chai SY, Fernando R, Peck G, Ye SY, Mendelsohn FA, Jenkins TA, Albiston AL (2004) The angiotensin IV/AT4 receptor. Cellular and molecular life sciences : CMLS 61 (21):2728-2737. 
487

488

489

490

491

492

493

494

495

496

497

498

499

500

501

502

503

504

505

506

507

doi:10.1007/s00018-004-4246-1

Chappell DL, McAvoy T, Weiss B, Weiner R, Laterza OF (2012) Development and validation of an ultra-sensitive method for the measurement of plasma renin activity in human plasma via LCMS/MS. Bioanalysis 4 (23):2843-2850. doi:10.4155/bio. 12.268

Chappell MC (2016) Biochemical evaluation of the renin-angiotensin system: the good, bad, and absolute? American journal of physiology Heart and circulatory physiology 310 (2):H137-152. doi:10.1152/ajpheart.00618.2015

Che J, Meng Q, Chen Z, Hou Y, Shan C, Cheng Y (2010) Quantitative analysis of a novel HIV fusion inhibitor (sifuvirtide) in HIV infected human plasma using high-performance liquid chromatography-electrospray ionization tandem mass spectrometry. Journal of pharmaceutical and biomedical analysis 51 (4):927-933. doi:10.1016/j.jpba.2009.10.018

Chertov O, Biragyn A, Kwak LW, Simpson JT, Boronina T, Hoang VM, Prieto DA, Conrads TP, Veenstra TD, Fisher RJ (2004) Organic solvent extraction of proteins and peptides from serum as an effective sample preparation for detection and identification of biomarkers by mass spectrometry. Proteomics 4 (4):1195-1203. doi:10.1002/pmic.200300677

Cima I, Schiess R, Wild P, Kaelin M, Schuffler P, Lange V, Picotti P, Ossola R, Templeton A, Schubert O, Fuchs T, Leippold T, Wyler S, Zehetner J, Jochum W, Buhmann J, Cerny T, Moch H, Gillessen S, Aebersold R, Krek W (2011) Cancer genetics-guided discovery of serum biomarker signatures for diagnosis and prognosis of prostate cancer. Proc Natl Acad Sci U S A 108 (8):3342-3347. doi:10.1073/pnas.1013699108

Cui L, Nithipatikom K, Campbell WB (2007) Simultaneous analysis of angiotensin peptides by 
508 LC-MS and LC-MS/MS: metabolism by bovine adrenal endothelial cells. Analytical 509 biochemistry 369 (1):27-33. doi:10.1016/j.ab.2007.06.045

510 Culver S, Li C, Siragy HM (2017) Intrarenal Angiotensin-Converting Enzyme: the Old and the

511 New. Current hypertension reports 19 (10):80. doi:10.1007/s11906-017-0778-2

512 Eliuk S, Makarov A (2015) Evolution of Orbitrap Mass Spectrometry Instrumentation. Annual 513 review of analytical chemistry 8:61-80. doi:10.1146/annurev-anchem-071114-040325

514 Finoulst I, Pinkse M, Van Dongen W, Verhaert P (2011a) Sample preparation techniques for the untargeted LC-MS-based discovery of peptides in complex biological matrices. Journal of biomedicine \& biotechnology 2011:245291. doi:10.1155/2011/245291

517 Finoulst I, Vink P, Rovers E, Pieterse M, Pinkse M, Bos E, Verhaert P (2011b) Identification of 518 low abundant secreted proteins and peptides from primary culture supernatants of human T-cells.

519 Journal of proteomics 75 (1):23-33. doi:10.1016/j.jprot.2011.03.034

520 Fyhrquist F, Saijonmaa O (2008) Renin-angiotensin system revisited. J Intern Med 264 (3):224236. doi:10.1111/j.1365-2796.2008.01981.x

522 Gallien S, Duriez E, Demeure K, Domon B (2013) Selectivity of LC-MS/MS analysis:

523 implication for proteomics experiments. Journal of proteomics 81:148-158. doi:10.1016/j.jprot.2012.11.005

Hu X, Kamberi M (2009) Quantification of apolipoprotein A-I mimetic peptide D-4F in rabbit plasma by high-performance liquid chromatography coupled with electrospray ionization tandem mass spectrometry. Journal of chromatography B, Analytical technologies in the biomedical and life sciences 877 (20-21):1946-1950. doi:10.1016/j.jchromb.2009.04.044 
529 Jackman HL, Massad MG, Sekosan M, Tan F, Brovkovych V, Marcic BM, Erdos EG (2002)

530 Angiotensin 1-9 and 1-7 release in human heart: role of cathepsin A. Hypertension 39 (5):976-

531 981. doi:10.1161/01.hyp.0000017283.67962.02

532 Jankowski V, Tolle M, Santos RA, Gunthner T, Krause E, Beyermann M, Welker P, Bader M, 533 Pinheiro SV, Sampaio WO, Lautner R, Kretschmer A, van der Giet M, Zidek W, Jankowski J 534 (2011) Angioprotectin: an angiotensin II-like peptide causing vasodilatory effects. FASEB 535 journal : official publication of the Federation of American Societies for Experimental Biology 25 (9):2987-2995. doi:10.1096/fj.11-185470

537 Kay RG, Challis BG, Casey RT, Roberts GP, Meek CL, Reimann F, Gribble FM (2018)

538 Peptidomic analysis of endogenous plasma peptides from patients with pancreatic 539 neuroendocrine tumours. Rapid communications in mass spectrometry : RCM 32 (16):1414540 1424. doi: $10.1002 / \mathrm{rcm} .8183$

541 Kemp BA, Bell JF, Rottkamp DM, Howell NL, Shao W, Navar LG, Padia SH, Carey RM (2012)

542 Intrarenal angiotensin III is the predominant agonist for proximal tubule angiotensin type 2 543 receptors. Hypertension 60 (2):387-395. doi:10.1161/HYPERTENSIONAHA.112.191403

544 Kiyonami R, Schoen A, Prakash A, Peterman S, Zabrouskov V, Picotti P, Aebersold R, Huhmer 545 A, Domon B (2011) Increased selectivity, analytical precision, and throughput in targeted 546 proteomics. Mol Cell Proteomics 10 (2):M110 002931. doi:10.1074/mcp.M110.002931

547 Lortie M, Bark S, Blantz R, Hook V (2009) Detecting low-abundance vasoactive peptides in 548 plasma: progress toward absolute quantitation using nano liquid chromatography-mass 549 spectrometry. Analytical biochemistry 394 (2):164-170. doi:10.1016/j.ab.2009.07.021 
550 Malchow S, Loosse C, Sickmann A, Lorenz C (2017) Quantification of Cardiovascular Disease

551 Biomarkers in Human Platelets by Targeted Mass Spectrometry. Proteomes 5 (4).

552 doi:10.3390/proteomes5040031

553 Morris DL, Sanghavi D, Kahwaji CI (2020) Angiotensin II. In: StatPearls. Treasure Island (FL),

555 Nachman RL, Jaffe EA (2004) Endothelial cell culture: beginnings of modern vascular biology.

556 The Journal of clinical investigation 114 (8):1037-1040. doi:10.1172/JCI23284

557 Olkowicz M, Chlopicki S, Smolenski RT (2017) A Primer to Angiotensin Peptide Isolation,

558 Stability, and Analysis by Nano-Liquid Chromatography with Mass Detection. Methods in

559 molecular biology 1614:175-187. doi:10.1007/978-1-4939-7030-8_14

560 Olkowicz M, Radulska A, Suraj J, Kij A, Walczak M, Chlopicki S, Smolenski RT (2015)

561 Development of a sensitive, accurate and robust liquid chromatography/mass spectrometric

562 method for profiling of angiotensin peptides in plasma and its application for atherosclerotic

563 mice. Journal of chromatography A 1393:37-46. doi:10.1016/j.chroma.2015.03.012

564 Ronsein GE, Pamir N, von Haller PD, Kim DS, Oda MN, Jarvik GP, Vaisar T, Heinecke JW

565 (2015) Parallel reaction monitoring (PRM) and selected reaction monitoring (SRM) exhibit

566 comparable linearity, dynamic range and precision for targeted quantitative HDL proteomics.

567 Journal of proteomics 113:388-399. doi:10.1016/j.jprot.2014.10.017

568 Santos RA, Ferreira AJ (2007) Angiotensin-(1-7) and the renin-angiotensin system. Curr Opin

569 Nephrol Hypertens 16 (2):122-128. doi:10.1097/MNH.0b013e328031f362

570 Sherman J, McKay MJ, Ashman K, Molloy MP (2009) How specific is my SRM?: The issue of 
571 precursor and product ion redundancy. Proteomics 9 (5):1120-1123.

572 doi:10.1002/pmic. 200800577

573 Stejskal K, Potesil D, Zdrahal Z (2013) Suppression of peptide sample losses in autosampler

574 vials. Journal of proteome research 12 (6):3057-3062. doi:10.1021/pr400183v

575 Streatfeild-James RM, Williamson D, Pike RN, Tewksbury D, Carrell RW, Coughlin PB (1998)

576 Angiotensinogen cleavage by renin: importance of a structurally constrained N-terminus. FEBS

577 letters 436 (2):267-270. doi:10.1016/s0014-5793(98)01145-4

578 Suski M, Gebska A, Olszanecki R, Stachowicz A, Uracz D, Madej J, Korbut R (2014) Influence

579 of atorvastatin on angiotensin I metabolism in resting and TNF-alpha -activated rat vascular

580 smooth muscle cells. Journal of the renin-angiotensin-aldosterone system : JRAAS 15 (4):378-

581 383. doi:10.1177/1470320313475907

582 Tamvakopoulos C (2007) Mass spectrometry for the quantification of bioactive peptides in

583 biological fluids. Mass spectrometry reviews 26 (3):389-402. doi:10.1002/mas.20120

584 Touyz RM (2003) The role of angiotensin II in regulating vascular structural and functional

585 changes in hypertension. Curr Hypertens Rep 5 (2):155-164

586 Tyrankiewicz U, Olkowicz M, Skorka T, Jablonska M, Orzylowska A, Bar A, Gonet M, 587 Berkowicz P, Jasinski K, Zoladz JA, Smolenski RT, Chlopicki S (2018) Activation pattern of 588 ACE2/Ang-(1-7) and ACE/Ang II pathway in course of heart failure assessed by multiparametric 589 MRI in vivo in Tgalphaq*44 mice. Journal of applied physiology 124 (1):52-65. 590 doi:10.1152/japplphysiol.00571.2017

591 Yu L, Yuan K, Phuong HT, Park BM, Kim SH (2016) Angiotensin-(1-5), an active mediator of 
592 renin-angiotensin system, stimulates ANP secretion via Mas receptor. Peptides 86:33-41.

593 doi:10.1016/j.peptides.2016.09.009

594 Yugandhar VG, Clark MA (2013) Angiotensin III: a physiological relevant peptide of the renin

595 angiotensin system. Peptides 46:26-32. doi:10.1016/j.peptides.2013.04.014

596 Zhong J, Basu R, Guo D, Chow FL, Byrns S, Schuster M, Loibner H, Wang XH, Penninger JM,

597 Kassiri Z, Oudit GY (2010) Angiotensin-converting enzyme 2 suppresses pathological

598 hypertrophy, myocardial fibrosis, and cardiac dysfunction. Circulation 122 (7):717-728, 718 p

599 following 728. doi:10.1161/CIRCULATIONAHA.110.955369 
602

603

604

605

606

607

608

609

610

611

612

613

614

615

616

617

618

619

620

\section{Figure legends}

Figure 1. Optimization of the LCMS/MS parameters. Chromatogram of the seven target peptides and internal standard peptide by LC-MS/MS. The seven target peptides and the internal standard peptide could complete the column elution with acetonitrile concentrations of $10 \%$ $16 \%$. In order to separate the elution peaks and not affect the peak shape, the separation stages were set using a gradient from 4\% B at 2 min to $22 \%$ B at 47 min. It can be seen that the peaks of each target peptide and internal standard peptide were smooth and effectively separated.

Figure 2. Establishment of the PRM detection model. The ion dwell time for Ang I, Ang (19), Ang II, and Ang (1-7) was adjusted according to the number of precursor ions. Using the peptide map library pre-built using Skyline, the fragment ions in the PRM scan data were extracted. The fracture law and abundance of fragment ions were matched with the spectrum library. The mass spectral response was up to $10^{6}$ orders of magnitude. The signal-to-noise ratio was high, and the mass spectral peak shape was smooth.

Figure 3. Determination of the sample stability over time. Stability (\% of changes in concentration) of the seven angiotensin peptides kept in the injector at $7^{\circ} \mathrm{C}$ over $8 \mathrm{~h}$.

\section{Figure 4. Determination of the number of sample loading on Ang detection. Evaluation of} the number of sample passages through the reverse C18 solid-phase extraction column packing on the recovery rate of angiotensin peptides. We used three loadings on the cartridge as control and evaluated the other numbers of loading times on the recovery rate of Ang by comparing the 
621 peak area ratio of the same target peptide quantitative fragment ion. The control group used three

622 passages.

623 Figure 5. Determination of the impact of ultrafiltration on sample recovery. Recovery rate

624 of angiotensin peptides after ultrafiltration and solid-phase extraction compared with solid-phase

625 extraction alone. ${ }^{* *} \mathrm{P}<0.01 . * * * \mathrm{P}<0.001$

626 Figure 6. ACE protein expression was successfully silenced in HUVECs. Western blotting

627 showing that the expression of the ACE protein in the ACE-shRNA-1 and ACE-shRNA-2

628 groups was significantly reduced (95.6\% and 78.2\%, respectively) compared with the PLKO.1

629 group. $* * \mathrm{P}<0.01 . * * * \mathrm{P}<0.001$

630 Figure 7. ACE silencing induces differences in the Ang metabolic profile that can be

631 detected by LC-MS/MS. To verify the practicability of the Ang assay established in this study,

632 Ang I was added at a final concentration of $1 \mu \mathrm{M}$ to three groups of HUVECs displaying

633 different levels of ACE expression. After incubation for $1 \mathrm{~h}$, Ang I and its major metabolites

634 (Ang II, Ang (1-7) and Ang (1-5), Ang (1-9), Ang III and Ang IV) were detected by the nano-

635 LC-MS/MS method established in this study. ${ }^{*} \mathrm{P}<0.05 . * * \mathrm{P}<0.01$. $* * * \mathrm{P}<0.001$.

636 
Figure 1

Optimization of the LCMS/MS parameters

Chromatogram of the seven target peptides and internal standard peptide by LC-MS/MS. The seven target peptides and the internal standard peptide could complete the column elution with acetonitrile concentrations of $10 \%-16 \%$. In order to separate the elution peaks and not affect the peak shape, the separation stages were set using a gradient from $4 \%$ B at 2 min to $22 \% \mathrm{~B}$ at $47 \mathrm{~min}$. It can be seen that the peaks of each target peptide and internal standard peptide were smooth and effectively separated.

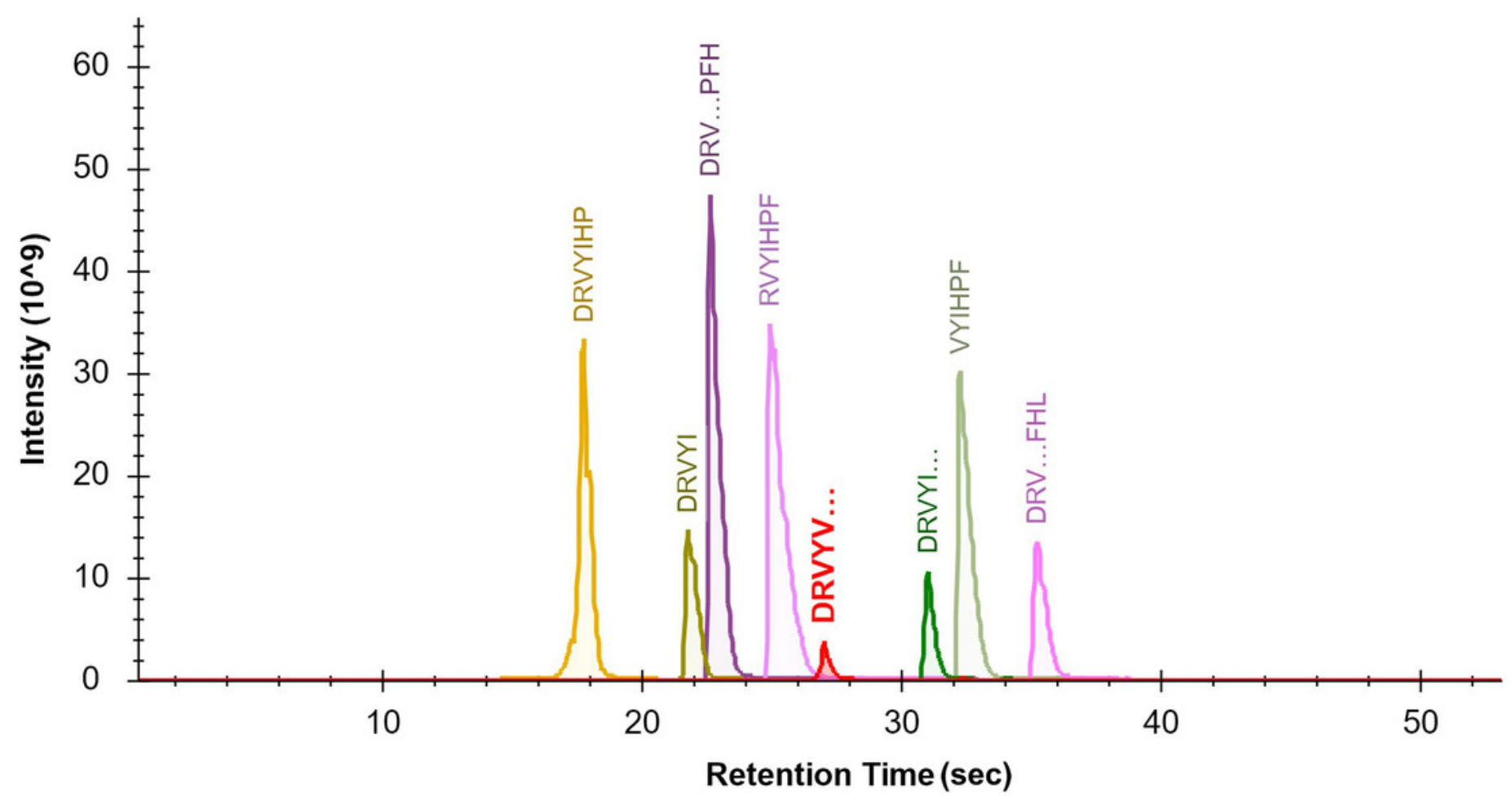




\section{Figure 2}

Establishment of the PRM detection model.

The ion dwell time for Ang I, Ang (1-9), Ang II, and Ang (1-7) was adjusted according to the number of precursor ions. Using the peptide map library pre-built using Skyline, the fragment ions in the PRM scan data were extracted. The fracture law and abundance of fragment ions were matched with the spectrum library. The mass spectral response was up to $10^{6}$ orders of magnitude. The signal-to-noise ratio was high, and the mass spectral peak shape was smooth. 

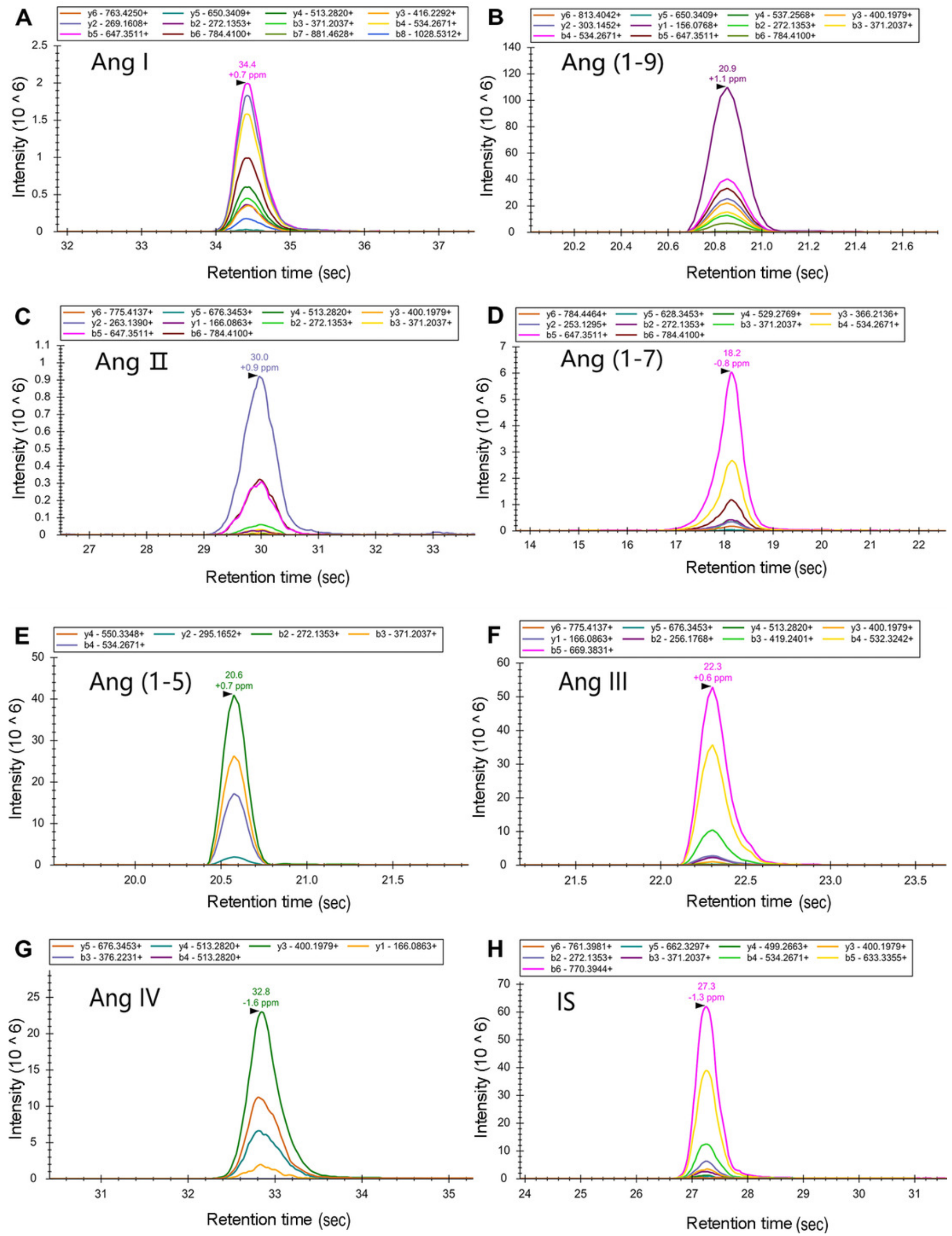
Figure 3

Determination of the sample stability over time

Stability (\% of changes in concentration) of the seven angiotensin peptides kept in the injector at $7^{\circ} \mathrm{C}$ over $8 \mathrm{~h}$.

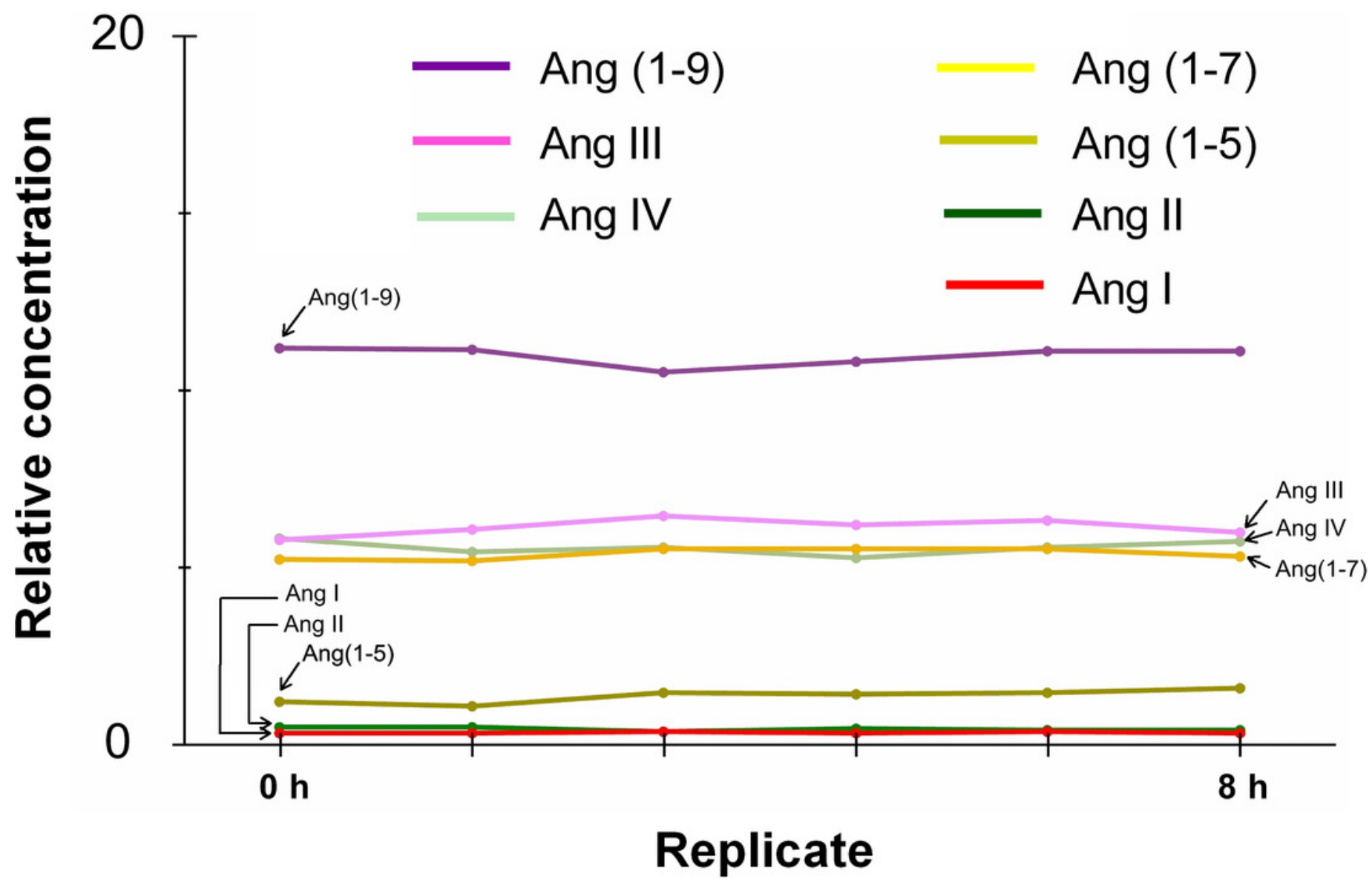


Figure 4

Determination of the number of sample loading on Ang detection

Evaluation of the number of sample passages through the reverse $\mathrm{C} 18$ solid-phase extraction column packing on the recovery rate of angiotensin peptides. We used three loadings on the cartridge as control and evaluated the other numbers of loading times on the recovery rate of Ang by comparing the peak area ratio of the same target peptide quantitative fragment ion. The control group used three passages.

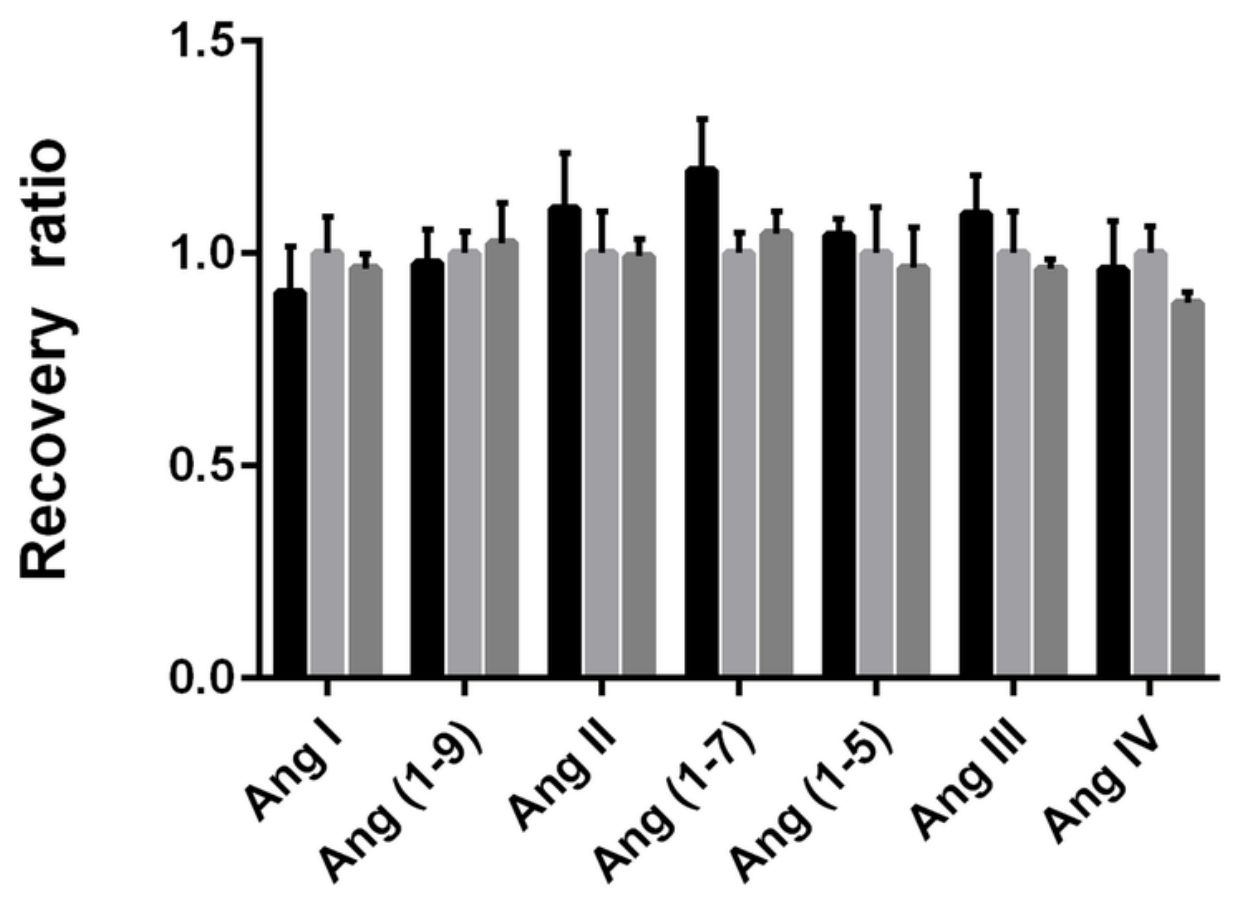

Loading 2 times Loading 3 times Loading 4 times 
Figure 5

Determination of the impact of ultrafiltration on sample recovery

Recovery rate of angiotensin peptides after ultrafiltration and solid-phase extraction compared with solid-phase extraction alone. ${ }^{*} \mathrm{P}<0.01$. ${ }^{* * * P}<0.001$.

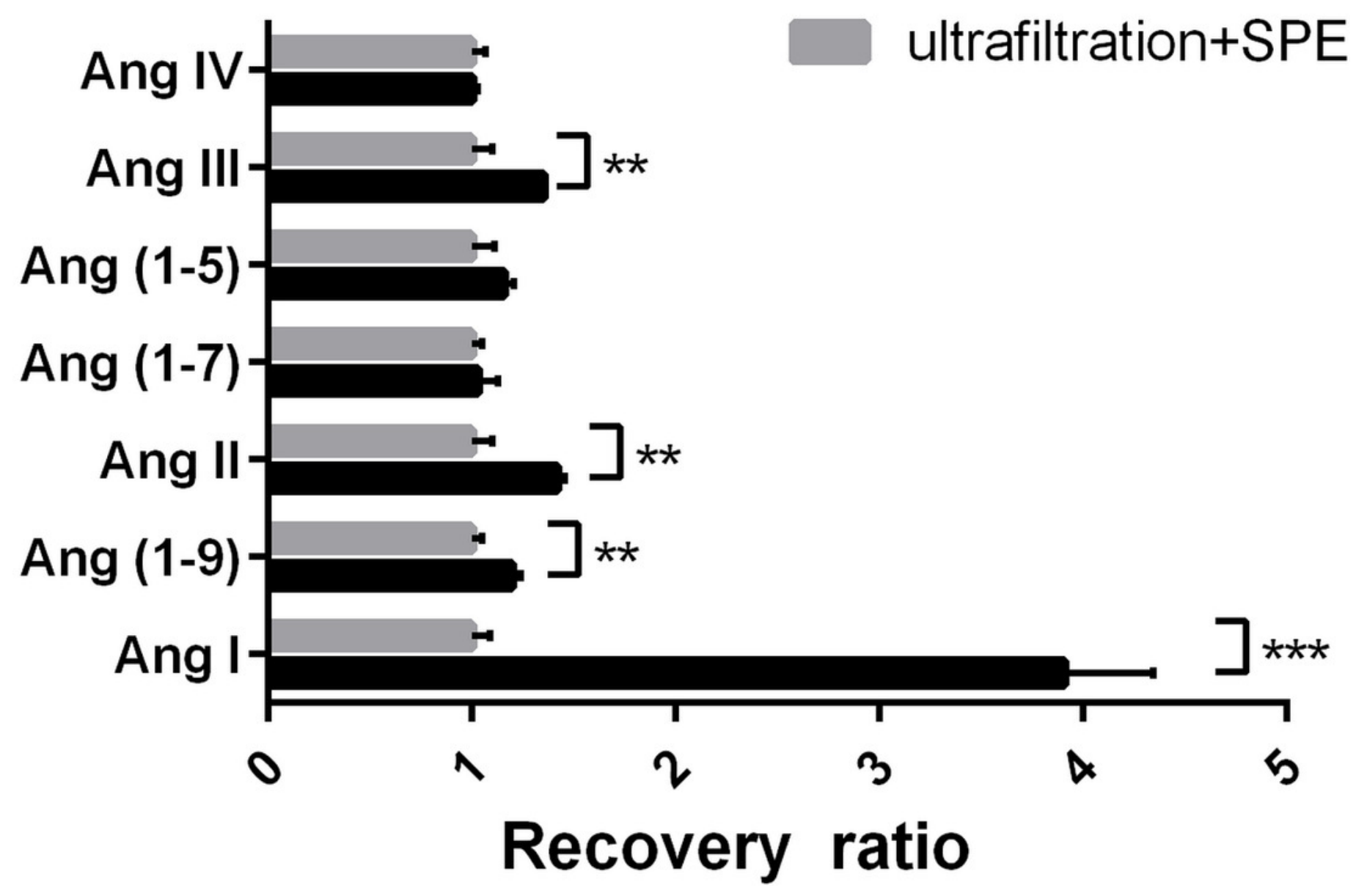


Figure 6

ACE protein expression was successfully silenced in HUVECS

Western blotting showing that the expression of the ACE protein in the ACE-shRNA-1 and ACE-shRNA-2 groups was significantly reduced (95.6\% and $78.2 \%$, respectively) compared with the PLKO.1 group. ${ }^{*} \mathrm{P}<0.01 . * * * \mathrm{P}<0.001$.

A

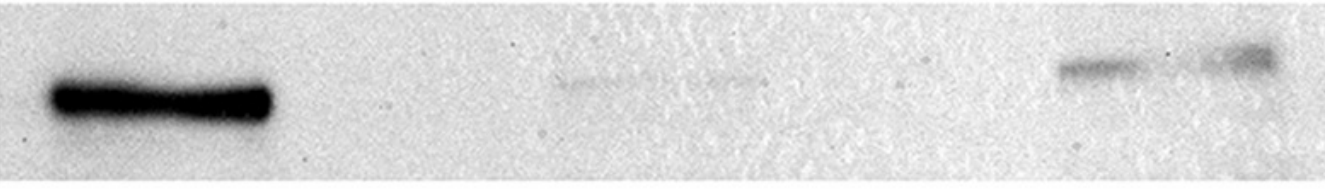

ACE

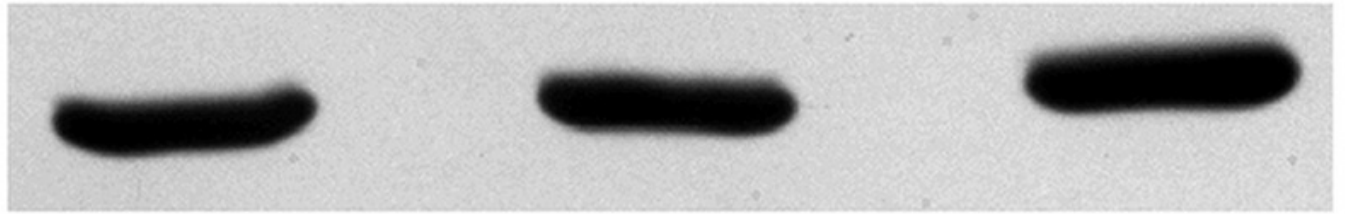

$\beta$ - actin

PLKO. 1 (NC) ACE-shRNA-1 ACE-shRNA- 2

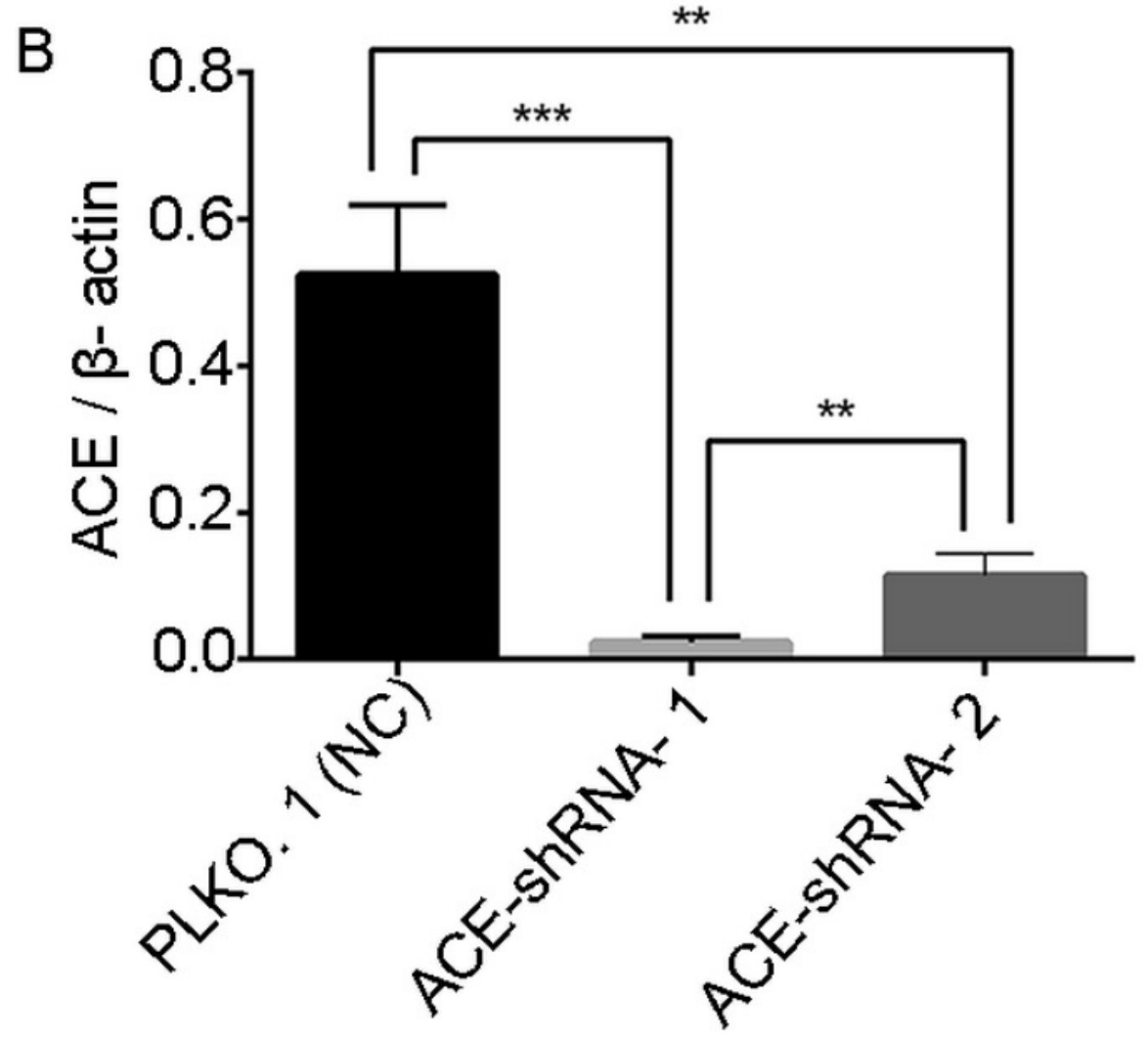

- PLKO. 1 (NC)

ACE-ShRNA- 1

$\square$ ACE-ShRNA- 2 


\section{Figure 7}

ACE silencing induces differences in the Ang metabolic profile that can be detected by LC-MS/MS

To verify the practicability of the Ang assay established in this study, Ang I was added at a final concentration of $1 \mu \mathrm{M}$ to three groups of HUVECs displaying different levels of ACE expression. After incubation for $1 \mathrm{~h}$, Ang I and its major metabolites (Ang II, Ang (1-7) and Ang (1-5), Ang (1-9), Ang III and Ang IV) were detected by the nano-LC-MS/MS method established in this study. $* P<0.05 . * * P<0.01$. ${ }^{* * * P}<0.001$. 
A

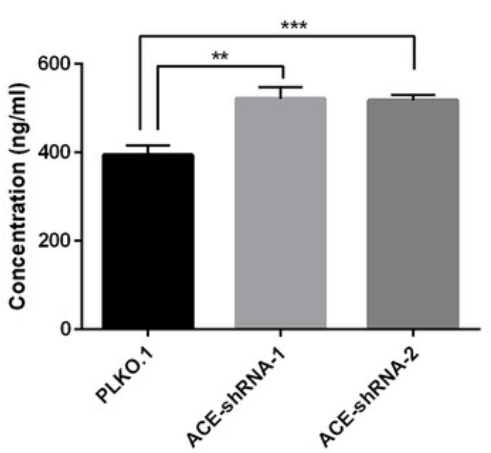

C

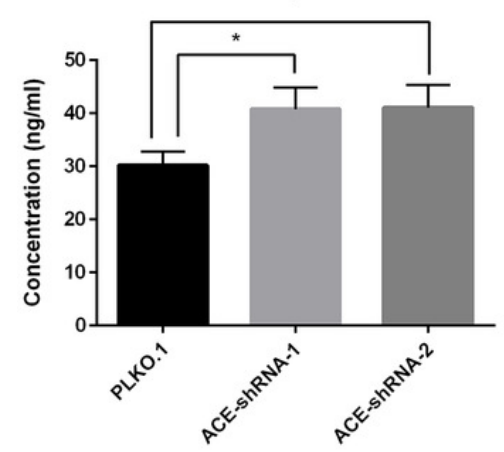

E

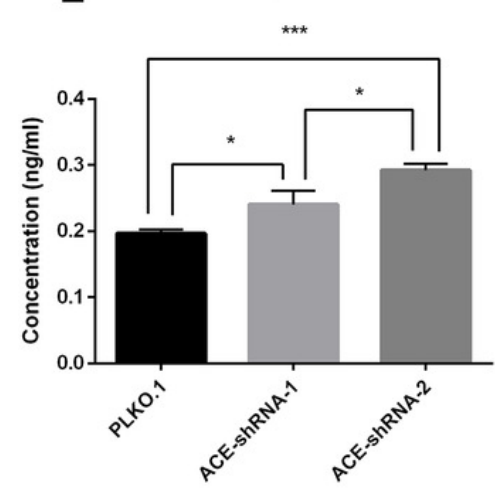

G

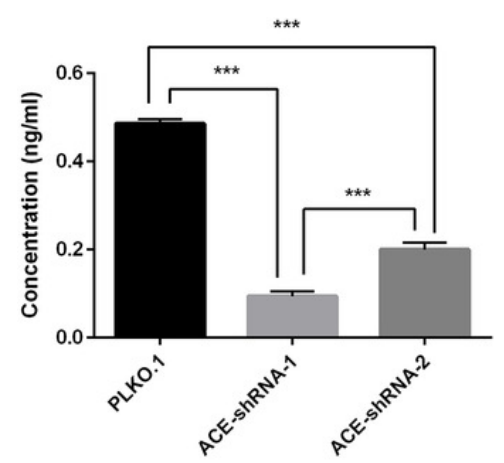

B

Ang II

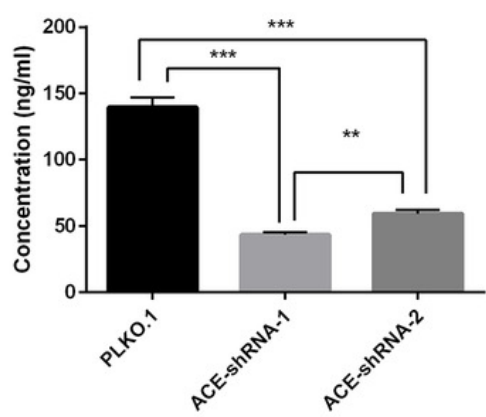

D

Ang (1-5)

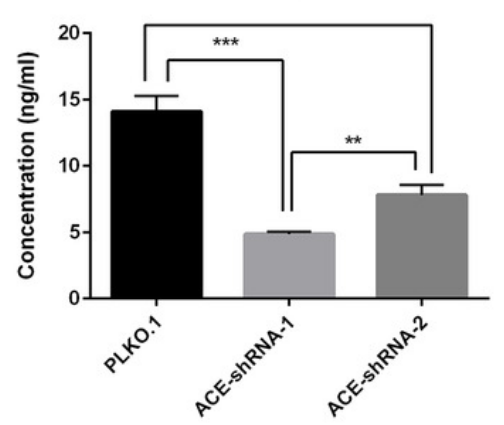

$\mathbf{F}$

Ang III

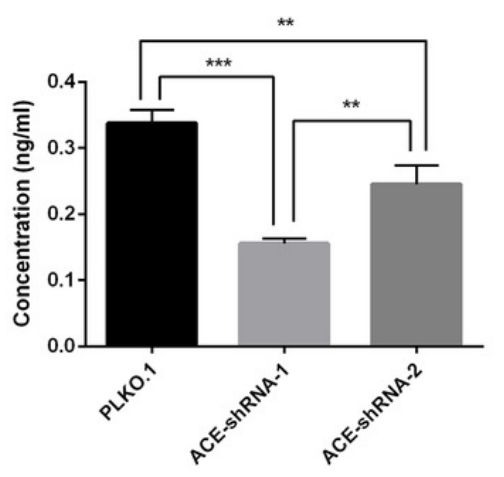

- PLKO.1

ACE-shRNA-1

- ACE-shRNA-2
ACE-shRNA-1

- ACE-shRNA-2
- PLKO.1

- ACE-shRNA-2

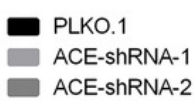

